\title{
Energetic Self-Optimization Induced by Stability in Low-Dissipation Heat Engines
}

\author{
J. Gonzalez-Ayala $\odot^{1,2}$ J. Guo,$^{3,1}$ A. Medina $\odot^{1,2}$ J. M. M. Roco๑ ${ }^{1,2}$ and A. Calvo Hernández $\odot^{1,2}$ \\ ${ }^{1}$ Departamento de Física Aplicada \\ ${ }^{2}$ Instituto Universitario de Física Fundamental y Matemáticas (IUFFyM), Universidad de Salamanca, 37008 Salamanca, Spain \\ ${ }^{3}$ College of Physics and Information Engineering, Fuzhou University, Fuzhou 350116, People's Republic of China
}

(Received 15 July 2019; accepted 14 January 2020; published 7 February 2020)

\begin{abstract}
The local stability of a weakly dissipative heat engine is analyzed and linked to an energetic multiobjective optimization perspective. This constitutes a novel issue in the unified study of cyclic energy converters, opening the perspective to the possibility that stability favors self-optimization of thermodynamic quantities including efficiency, power and entropy generation. To this end, a dynamics simulating the restitution forces, which mimics a harmonic potential, bringing the system back to the steady state is analyzed. It is shown that relaxation trajectories are not arbitrary but driven by the improvement of several energetic functions. Insights provided by the statistical behavior of consecutive random perturbations show that the irreversible behavior works as an attractor for the energetics of the system, while the endoreversible limit acts as an upper bound and the Pareto front as a global attractor. Fluctuations around the operation regime reveal a difference between the behavior coming from fast and slow relaxation trajectories: while the former are associated to an energetic self-optimization evolution, the latter are ascribed to better performances. The self-optimization induced by stability and the possible use of instabilities in the operation regime to improve the energetic performance might usher into new useful perspectives in the control of variables for real engines.
\end{abstract}

DOI: 10.1103/PhysRevLett.124.050603

Introduction.-The optimization of energy converters, particularly of heat engines (HE), is usually focused on relationships among the operational parameters of a system. As the panacea of thermodynamic optimization, the simultaneous optimization of all energetic functions is impossible; one must look for compromise solutions with given constraints. New hints reveal that fluctuations could be valuable insights in the optimization analysis when pushing the system to optimum power and efficiency altogether [1]. Moreover, the role of limited control on system operation variables is a challenging issue [2-4]. The appearance of trade-off relations involving efficiency, power, and constancy [5,6] suggests that there is an inherent energetic preference stemming from the restitution mechanism in charge of the HE stability and whether thermodynamical optimization underlies stability.

Due to the impossibility of obtaining the Carnot efficiency for irreversible heat devices and the high energetic cost of the maximum power regime [7-14], trade-off based figures of merit (including atop minimization of entropy as a desirable requirement) have played a key role in the optimization of heat devices. This is the case of the ecological [15], Omega [16], and efficient power [17] functions; see also [18-20]. Beside this, studies on the fluctuations of relevant energetic properties show subtle differences for quasistatic and steady state HE models providing different strategies to account for control of parameters and engine layouts in macroscopic, mesoscopic, and quantum frameworks [5,6,21-23]. Preliminary studies $[24,25]$ suggest a connection between stability around a steady state and the energetic self-optimization of cyclic HEs for figures of merit that balances power, entropy, and efficiency. Consequently, the evolution of the heat engine to a steady state could be itself an optimization strategy whenever facing a limitation in variables control. Here this is addressed for a weakly dissipative HE driven by dynamics simulating restitution forces that bring the system back to the steady state. The low-dissipation (LD) model [26] is a first order irreversible deviation from a Carnot cycle, independent of a specific heat transfer law. It focuses on the symmetries and asymmetries of the dissipations on the system while in contact with external heat baths, allowing for a general time-dependent formulation on entropy generation [27,28]. Its validity [29-33] comprises a broad temperature range and applications for macroscopic, mesoscopic, and microscopic models [8,30,33-40]. A stability dynamics linking variations on the heat exchanges and the contact times with the external baths around the stable point is assumed. Altogether, the model and the dynamics provide a suitable way to explore general behaviors not linked with particularities in the heat transfer mechanism but to time evolution.

These features are explored for maximum power (MP) and maximum omega $(\mathrm{M} \Omega)$, as a representative trade-off function. After a brief background of both regimes, the stability dynamics is analyzed and joined to the energetic 
optimization in the context of the Pareto front and the MP and $M \Omega$ regimes. Stochastic perturbations on the operation regimes are also explored together with the consequences on the energetic self-optimization. Finally, some perspectives are discussed.

The control variables. - The low-dissipation model considers irreversible deviations from a Carnot cycle working between two thermal reservoirs at constant temperatures $T_{c}$ and $T_{h}\left(>T_{c}\right)$ assuming finite contact times $\left\{t_{c}, t_{h}\right\}$ of each isothermal process (the duration of adiabatic processes is neglected). The information regarding irreversibilities in the contacts with each reservoir are accounted by the coefficients $\left\{\Sigma_{c}, \Sigma_{h}\right\}$. The total entropy change is $\Delta S_{\text {tot }}=$ $\Sigma_{c} / t_{c}+\Sigma_{h} / t_{h}$. The reversible limit is recovered whenever $\left\{t_{c}, t_{h}\right\} \rightarrow \infty$ or $\left\{\Sigma_{c}, \Sigma_{h}\right\} \rightarrow 0$. An useful description is achieved by means of the dimensionless variables $\tilde{t_{c}} \equiv t_{c} \Delta S / \Sigma_{h}, \tilde{t}_{h} \equiv t_{h} \Delta S / \Sigma_{h}$, accounting for the system size, and where $\Delta S$ is the entropy change at the hot isotherm of the baseline Carnot cycle. Expressing the energy in $T_{h} \Delta S$ units, the exchanged heats with the hot and cold reservoirs $Q_{h}$ and $Q_{c}$ are $\tilde{Q}_{c}=-\tau-\tau \Sigma / \tilde{t}_{c}$, and $\tilde{Q}_{h}={\tilde{t_{h}}}^{-1}-1$, where $\tau=T_{c} / T_{h}$ and $\Sigma \equiv \Sigma_{c} / \Sigma_{h}$. Similarly, the efficiency, $\eta=\left(Q_{h}+Q_{c}\right) / Q_{h}$, power output, $P=$ $\left(Q_{h}+Q_{c}\right) /\left(t_{c}+t_{h}\right)$, entropy production $\sigma=\Delta S_{\text {tot }} /\left(t_{c}+t_{h}\right)$ and $\Omega=\left(2 \eta-\eta_{C}\right) \dot{Q}_{h}$ [16] $\quad\left(\eta_{C}=1-\tau\right.$ is the Carnot efficiency) are given by the following:

$$
\begin{aligned}
& \tilde{P}=\frac{P}{T_{h} \Delta S}=\frac{\eta_{C}-\frac{1}{\tilde{t}_{h}}-\frac{\tau \Sigma}{\tilde{t}_{c}}}{t_{c}+t_{h}}, \quad \eta=\frac{\eta_{C}-\frac{1}{\tilde{t}_{h}}-\frac{\tau \Sigma}{\tilde{t}_{c}}}{1-\frac{1}{\tilde{t}_{h}}}, \\
& \tilde{\Omega}=\frac{\Omega}{T_{h} \Delta S}=\frac{\eta_{C}-\frac{1}{\tilde{t}_{h}}-\frac{2 \tau \Sigma}{\tilde{t}_{c}}}{\tilde{t}_{c}+\tilde{t}_{h}}, \quad \tilde{\sigma} \equiv \frac{\sigma}{\Delta S}=\frac{\Sigma \tau}{\tilde{t}_{c}}+\frac{1}{\tilde{t}_{h}} .
\end{aligned}
$$

All of them have a dependence on $\left\{\tau, \Sigma, \tilde{t}_{c}, \tilde{t}_{h}\right\}[28,31]$; $\left\{\tilde{t}_{c}, \tilde{t}_{h}\right\}$ for MP and M $\Omega$ conditions are as follows:

$\tilde{t}_{c}^{\mathrm{MP}}=\frac{2 \tau \Sigma\left(1+\frac{1}{\sqrt{\tau \Sigma}}\right)}{1-\tau}, \quad \tilde{t}_{c}^{\mathrm{M} \Omega}=\frac{4 \tau \Sigma\left(1+\frac{1+\tau}{\sqrt{2 \tau \Sigma}}\right)}{1-\tau}$,

$\tilde{t}_{h}^{\mathrm{MP}}=\frac{2(1+\sqrt{\tau \Sigma})}{1-\tau}, \quad \tilde{t}_{h}^{\mathrm{M} \Omega}=\frac{2(1+\tau)\left(1+\sqrt{\frac{2 \tau \Sigma}{1+\tau}}\right)}{1-\tau}$,

$\{\tau, \Sigma\}$ can be considered as fixed parameters. This allows to define total operation times $\tilde{t}_{\text {tot }}^{\mathrm{MP}} \equiv \tilde{t}_{c}^{\mathrm{MP}}+\tilde{t}_{h}^{\mathrm{MP}}$ and $\tilde{t}_{\mathrm{tot}}^{\mathrm{M} \Omega} \equiv$ $\tilde{t}_{c}^{\mathrm{M} \Omega}+\tilde{t}_{h}^{\mathrm{M} \Omega}$. Under time constraints parabolic and looplike $P$ vs $\eta$ curves (typical in endoreversible and irreversible models, respectively) are obtained $[28,41]$.

The best energetic performance: the Pareto front.There are no configurations fulfilling altogether the maximization of efficiency, power, $\Omega$, and entropy minimization. The multiobjective optimization problem involves the simultaneous maximization or minimization of a number of objective functions [42] and the so-called Pareto front gives

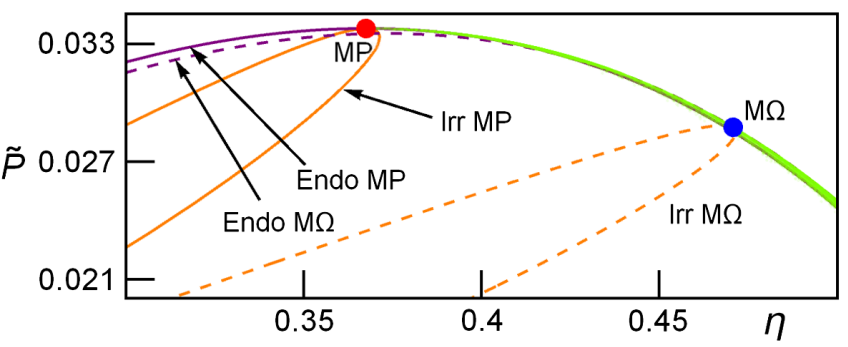

FIG. 1. Pareto front for the LD heat engine. Endoreversible and irreversible limits are depicted. Here $\tau=2 / 5$ and $\Sigma=1$. The introduced algorithm to obtain the Pareto front is addressed in more detail in the companion paper [41].

the best compromise among all of them. Thus, the Pareto front is obtained (Fig. 1), showing that the endoreversible limit is linked with the multiobjective optimization. The Kullback-Leibler divergence (KLD) [41,43] is used to assure statistical convergence.

Stability dynamics.-A dynamical evolution of the engine is now addressed. It will be considered a time evolution of the heats absorbed and released associated to a dimensionless dynamical time, $t$ (with a characteristic timescale to be chosen later). The steady state where the system, at fixed control variables, is working either at maximum power or maximum $\Omega$ represents an stable point with associated contact times $\tilde{t}_{x}^{*}$ where $x$ refers to $\{c, h\}$ and * to either MP or M $\Omega$ [Eqs. (3) and (4)]. Heat transfers at the steady state can be thus denoted as $\tilde{Q}_{x}\left(\tilde{t}_{x}^{*}\right)$. Within the LD model it is straightforward to assume that time evolution in heat transfers is implicitly associated to contact times. In other words, heat transfers evolve from one cycle to another because contact times evolve, i.e., $\tilde{Q}_{x}=\tilde{Q}_{x}\left(\tilde{t}_{x}\right)$ and $\tilde{t_{x}}=\tilde{t_{x}}(t)$. So, a small perturbation over the stationary regime leads to an evolution of heat transfers that can be considered as associated to an oscillatory behavior around a stable fixed point. The most simple evolution of contact times is given by the linear case $d\left(\tilde{t}_{x}-\tilde{t}_{x}^{*}\right) / d t \propto-\left(\tilde{t}_{x}-\tilde{t}_{x}^{*}\right)[41,44]$. Besides, a first order expansion of the heat is given by $\tilde{Q}_{x}\left(\tilde{t}_{x}\right)-\tilde{Q}_{x}\left(\tilde{t}_{x}^{*}\right)=$ $\left.\left[d \tilde{Q}_{x}\left(\tilde{t}_{x}\right) / d \tilde{t}_{x}\right]\right|_{\tilde{t}_{x}^{*}}\left(\tilde{t}_{x}-\tilde{t}_{x}^{*}\right)$. Allowing to formulate a dynamics of contact times in the form of

$$
\begin{gathered}
\frac{d \tilde{t}_{c}}{d t}=A\left(\tilde{Q}_{c}\left(\tilde{t}_{c}^{*}\right)-\tilde{Q}_{c}\left(\tilde{t_{c}}\right)\right), \\
\frac{d \tilde{t}_{h}}{d t}=B\left(\tilde{Q}_{h}\left(\tilde{t}_{h}^{*}\right)-\tilde{Q}_{h}\left(\tilde{t}_{h}\right)\right),
\end{gathered}
$$

where $\{A, B\}$ are positive constants giving the restitution strengths (the larger the system, the faster it returns to the steady state). In the linear approximation the solution is determined by the eigenvectors and the eigenvalues, $\left\{\lambda_{1}, \lambda_{2}\right\}$, of the corresponding Jacobian matrix (see [24]), related to the relaxation times by $t_{j} \equiv \lambda_{j}^{-1}$ so that 

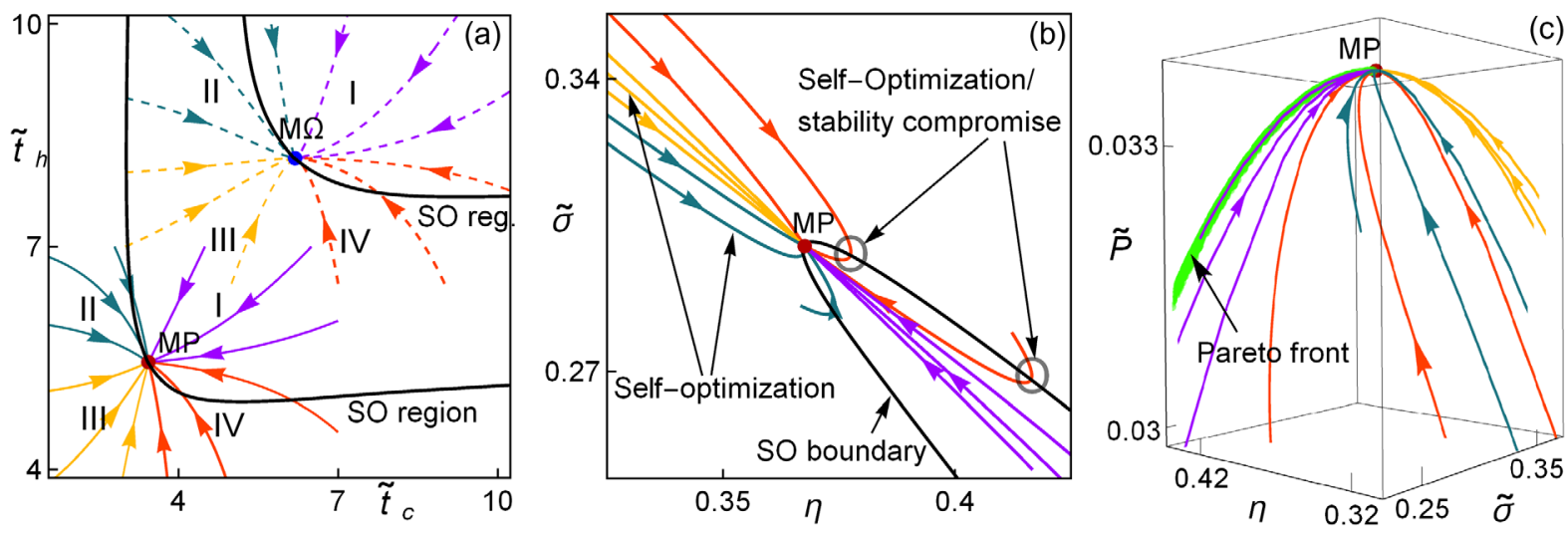

FIG. 2. (a) Trajectories according to Eqs. (5)-(6) in the phase space for MP and M $\Omega$. Corresponding trajectories for MP in the $\eta-\tilde{\sigma}$ plane (b) and in the $\eta-\tilde{P}-\tilde{\sigma}$ surface (c). Similar results are obtained for M $\Omega$ (see the companion paper [41]). Note the region in which stability induces a simultaneous optimization of $\eta$ and $\tilde{\sigma}$, delineated by the SO boundary. Note also the trend in the relaxation trajectories to approach the Pareto front (this feature is more obvious for the $\mathrm{M} \Omega$ regime). In all figures $\Sigma=1, \tau=2 / 5, A=B$, and $t_{\text {relax }}^{*}=\tilde{t}_{\text {tot }}^{*}$. The self-optimization and stability compromise is better pictured in the behavior change from optimization to a backwards evolution due to the need of the system to reach the steady state.

$$
\begin{gathered}
t_{i}^{\mathrm{MP}}=\frac{4}{\eta_{C}^{2} I_{i}}\left(\sqrt{\Sigma\left(1-\eta_{C}\right)}+1\right)^{2}, \\
t_{i}^{\mathrm{M} \Omega}=\frac{4}{\eta_{C}^{2} I_{i}}\left(\frac{\sqrt{2 \Sigma\left(1-\eta_{C}\right)}}{2-\eta_{C}}+1\right)^{2}\left(2-\eta_{C}\right),
\end{gathered}
$$

where $i=\{1,2\}, I_{1}=A$ and $I_{2}=B$. It is straightforward to express the relaxation time in terms of the operation time, i.e., $t_{\text {relax }}^{*} \equiv t_{1}^{*}+t_{2}^{*}=\left(2 / \eta_{C}\right)\left(A^{-1}+B^{-1}\right) \tilde{t}_{\text {tot }}^{*}$, connecting optimization and stability. In a cyclic process the system should return to the steady state within a cycle, constraining $\{A, B\}$ to $A^{-1}+B^{-1} \leq \eta_{C} / 2$.

Figure 2(a) shows in the phase space the location of the two stable points (MP and M $\Omega$ ) and some representative relaxation trajectories following the dynamics defined in Eqs. (5) and (6), with different colors for each quadrant (I-IV). Figure 2(b) shows their corresponding behaviors in the $\eta$ vs $\tilde{\sigma}$ plane. In this plane the trajectories tend to evolve in a narrow zone: while those in quadrants I (purple color) and III (yellow) arrive to the steady state in a direct way, those in quadrants II (cyan) and IV (orange) get the steady state after more or less abrupt turns. In Fig. 2(c) the behavior of $\tilde{P}$ is incorporated in a $3 \mathrm{D}$ view showing the location of the Pareto front. The trajectories evolving straight to the steady state are located in a small neighborhood of the Pareto front.

In Fig. 2 two kinds of trajectories stand out. Some with a simultaneous improvement of $\eta, \tilde{P}$, and $\tilde{\sigma}$. This behavior is enclosed in the so-called self-optimization (SO) region, bounded by the SO curve (black color in Fig. 2). Outside, the relaxation to the steady state exhibits another behavior with either an increasing of entropy or a decreasing in efficiency or power output. In the phase space the characterization of the SO boundary is given by the relaxation velocity $v_{\mathrm{dyn}}=\left[\left(d \tilde{t}_{c} / d t\right)^{2}+\left(d \tilde{t}_{h} / d t\right)^{2}\right]^{1 / 2}$. When $d v_{\mathrm{dyn}} / d t$ reaches certain values $c_{1}$ or $c_{2}$ (for the horizontal or vertical branches) in the energetic space there is an inflection behavior [see Fig. 2(b)]. More details can be found in the companion paper [41]. Figure 3, displays constant velocities contours, showing that the MP regime exhibits a faster evolution to the stable point than the $\mathrm{M} \Omega$ regime. For both regimes the fastest transitions mostly occur in quadrant III.

By gathering the information from the phase and energetic spaces a kind of "preference" on fast trajectories to improve their performance is exhibited. If the system is already in a "better" state, the relaxation is slower.

Random perturbations on the operation regime.-Now it is assumed that the system undergoes $N$ consecutive perturbations equally distributed in time along one cycle in subintervals of length $\Delta t$. The $N$ steps are computed by solving the stochastic differential equation based on Eqs. (5) and (6), using normally distributed random variables

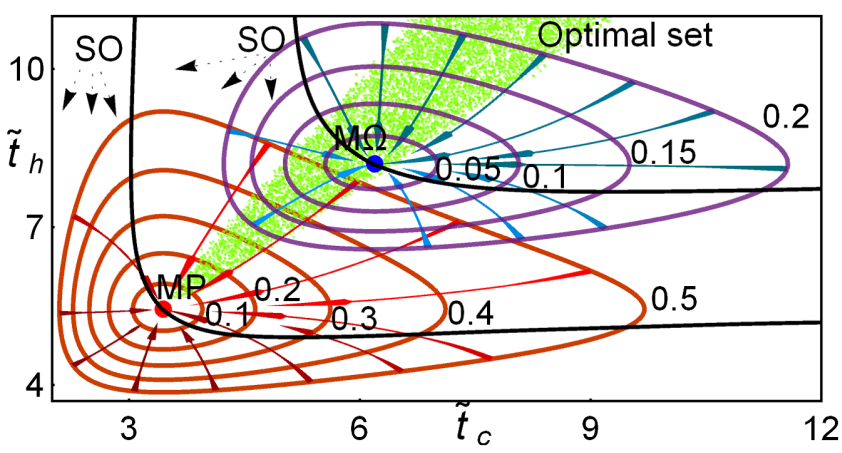

FIG. 3. Representative stream plot of the velocity field. Dynamical velocity contours show the speed in the relaxation toward the steady states. $\tau=2 / 5, \Sigma=1$ and $t_{\text {relax }}^{*}=\tilde{t}_{\text {tot }}^{*}$. 

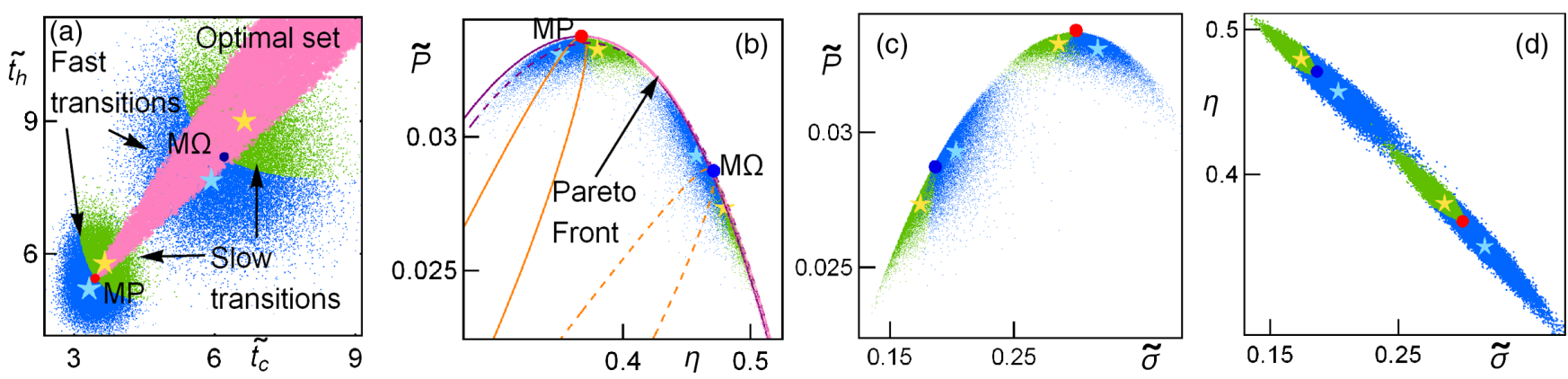

FIG. 4. (a) Average states for $5 \times 10^{4}$ cycles [green (blue) points are for those states outside (inside) the self-optimization region] and the Pareto front. Yellow and blue stars are the geometric center of the points inside each region; accordingly, blue points are closer to the steady state in the phase space. Behaviors of $\tilde{P}$ vs $\eta, \tilde{P}$ vs $\sigma$ and $\eta$ vs $\sigma$ are depicted in (b), (c), and (d), respectively. $N=10^{4}$, $\sigma_{\alpha}=\alpha^{*} / 10, \sigma_{\tilde{t}}=\tilde{t}^{*} / 10, A=B=4 /(1-\tau)$ so $t_{\text {relax }}^{*}=\tilde{t}_{\mathrm{tot}}^{*}, \tau=2 / 5$, and $\Sigma=1$.

following a two-dimensional Gaussian distribution, $\left\{\xi_{1}, \xi_{2}\right\}$, in the $\left\{\tilde{t}_{c}, \tilde{t}_{h}\right\}$ directions, as additive white noise by means of the Euler-Maruyama method [45]

$$
\begin{aligned}
& \Delta \tilde{t}_{c_{i}}=A\left(\tilde{Q}_{c}\left(\tilde{t}_{c}^{*}, \tilde{t}_{h}{ }^{*}\right)-\tilde{Q}_{c}\left(\tilde{t_{c_{i}}}, \tilde{t_{h_{i}}}\right)\right) \Delta t+\xi_{1} \sqrt{\Delta t}, \\
& \Delta \tilde{t}_{h_{i}}=B\left(\tilde{Q}_{h}\left(\tilde{t_{c}},{\tilde{t_{h}}}^{*}\right)-\tilde{Q}_{h}\left(\tilde{t_{c_{i}}}, \tilde{t_{h_{i}}}\right)\right) \Delta t+\xi_{2} \sqrt{\Delta t},
\end{aligned}
$$

where $\xi_{1}$ and $\xi_{2}$ follow the Gaussian distribution

$$
f_{\xi}\left(\tilde{t}_{c}, \tilde{t}_{h}\right)=\frac{\beta^{2}}{2 \pi \tilde{t}_{c}^{*} \tilde{t}_{h}^{*}} e^{\left.-\beta^{2} / 2\left[\tilde{t}_{c} / \tilde{t}_{c}^{*}\right)^{2}+\left(\tilde{t}_{h} / \tilde{t}_{h}^{*}\right)^{2}\right]}
$$

and $\beta$ is such that the standard deviations are given by $\sigma_{\tilde{t}_{c}}=\tilde{t}_{c}^{*} / \beta$ and $\sigma_{\tilde{t}_{h}}=\tilde{t}_{h}^{*} / \beta$. The initial state is $\left(\tilde{t}_{c_{1}}, \tilde{t}_{h_{1}}\right)=$ $\left(\tilde{t}_{c}{ }^{*}, \tilde{t}_{h}{ }^{*}\right) ; \Delta t$ is $\tilde{t}_{\text {tot }}^{*} / N$, so that after $N$ steps one cycle is fulfilled. After one cycle has been completed, the system starts another random trajectory from the steady state, without any information regarding previous cycles.

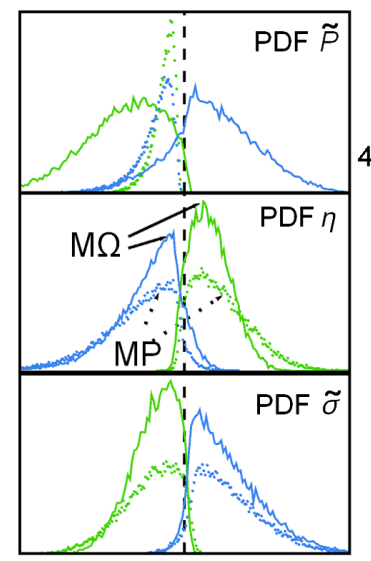

Steady State

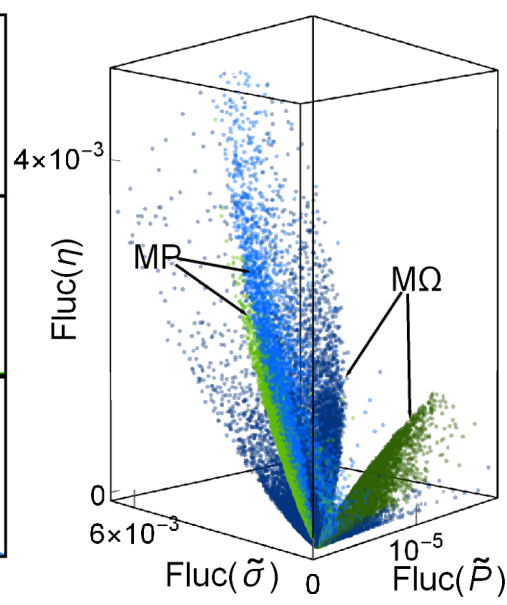

FIG. 5. (a) Probability distribution functions of the averages of $\tilde{P}, \eta$, and $\tilde{\sigma}$. Distributions for MP (dots) and M $\Omega$ (solid) are superposed centered in the steady state. Blue (green) stands for averages in the self-optimization (better-performance) regions. (b) Fluctuations around the steady state.
This procedure is repeated for $5 \times 10^{4}$ cycles. Statistical convergence is tested using the Kullback-Leibler divergence of the system energetic distributions [41].

The averaged loci in the phase space for each cycle are shown in Fig. 4(a), together with the Pareto optimal set. States within the region of better performance (green color, slow transitions) and of self-optimization (blue, fast transitions) are distinguished. Faster transitions keep the system closer to the steady state (see stars indicating the geometric center of points in each region). The states with a better performance are less spread out than those of rapid relaxation and they comprise the $47 \%$ of the cases for MP and $44.3 \%$ for $\mathrm{M} \Omega$; i.e., there is a larger density of average states in the better performance zone. In Figs. 4(b)-4(d) the corresponding averages of $\tilde{P}, \tilde{\sigma}$, and $\eta$ are shown. For both operation regimes the states of better performance (green points) are closer to the Pareto front, with notorious smaller variations in efficiency and an overall performance with higher efficiency and smaller entropy production.

Deviations from the steady state are illustrated by the probability distribution function (PDF) of $\tilde{P}, \eta$ and $\tilde{\sigma}$ [Fig. 5(a)]. The MP case has a sharper profile in $\tilde{P}$ (larger concentration of points near the steady state) but a larger dispersion in $\eta$ and $\tilde{\sigma}$ with respect to the $\mathrm{M} \Omega$ regime. Figure 5(b) shows the fluctuations around the steady state, given by $\operatorname{Fluc}(\phi)=\left(\phi^{*}-\langle\phi\rangle\right)^{2}$, where \langle\rangle is the average over a cycle. Fluctuations in the self-optimization zone for $\mathrm{M} \Omega$ are larger for $\tilde{P}$ but smaller for $\eta$ and $\tilde{\sigma}$ compared with the MP case. Those on $\tilde{\sigma}$ and $\eta$ are of the same order in both regimes with a sharper profile and slightly lesser entropy production in the $\mathrm{M} \Omega$ case.

Concluding remarks and perspectives.-It is shown that local stability of an operation regime around a steady state can be analyzed by heat exchange perturbations that induce an energetic trade-off self-optimization mechanism according to the velocity of trajectories and in agreement with results from a multiobjective optimization. The presented results are general in the weakly dissipative regime since the LD framework focuses in the relation between time 
constraints and entropy production. The equivalence of LD heat devices with many other cyclic and noncyclic models at very different scales [29-33] could provide a path to explore these behaviors when the particularities of the heat exchanges are considered. In such cases the dynamical equations will lastly depend on the particular operation variables. Some representative examples are (i) a macroscopic solarized irreversible Brayton engine with fluctuations induced by variable solar irradiance [46], (ii) a LD micrometric HE experimentally realized by a single particle in an optical trap through an optical harmonic potential $[47,48]$, and (iii) a spin vortex confined on a disc with a harmonic potential $[49,50]$.

In the companion paper [41], one different dynamic yielding a basin of attraction (instead of an stable equilibrium point) is also analyzed. A possible perspective of this work is its incorporation into the thermodynamic price of control analysis, where the self-optimization induced by a limited control could be useful. Finally, in the used multiobjective optimization all functions are equally weighted but a reverse study could be made. For a given local stability in natural systems, an inference on a different trade-off with specific weights could be an interesting path towards the analysis of natural optimization requirements [51-56]. This could lead to a hierarchy in the objective functions during evolutionary adaptation in specialized systems [57]. The role of analysis like the one performed here in the trade-off between power, efficiency, and constancy $[5,6]$ as the system approaches $\eta_{C}$ requires a further study as it is not clear if external perturbations could reveal intrinsic properties of constancy.

The authors acknowledge financial support from University of Salamanca Contract No. 2017/X005/1, Junta de Castilla y León Project No. SA017P17, and the National Natural Science Foundation of China (No. 11405032).

[1] D. Bercioux, R. Egger, P. Hnggi, and M. Thorwart, Focus on nonequilibrium fluctuation relations: From classical to quantum, New J. Phys. 17, 020201 (2015).

[2] J. M. Horowitz and K. Jacobs, Energy Cost of Controlling Mesoscopic Quantum Systems, Phys. Rev. Lett. 115, 130501 (2015).

[3] B. B. Machta, Dissipation Bound for Thermodynamic Control, Phys. Rev. Lett. 115, 260603 (2015).

[4] A.C. Barato and U. Seifert, Thermodynamic cost of external control, New J. Phys. 19, 073021 (2017).

[5] P. Pietzonka and U. Seifert, Universal Trade-Off between Power, Efficiency, and Constancy in Steady-State Heat Engines, Phys. Rev. Lett. 120, 190602 (2018).

[6] V. Holubec and A. Ryabov, Cycling Tames Power Fluctuations Near Optimum Efficiency, Phys. Rev. Lett. 121, 120601 (2018).

[7] F. L. Curzon and B. Ahlborn, Efficiency of a Carnot engine at maximum power output, Am. J. Phys. 43, 22 (1975).
[8] T. Schmiedl and U. Seifert, Efficiency of molecular motors at maximum power, Europhys. Lett. 83, 30005 (2008).

[9] C. Van den Broeck, N. Kumar, and K. Lindenberg, Efficiency of Isothermal Molecular Machines at Maximum Power, Phys. Rev. Lett. 108, 210602 (2012).

[10] N. Golubeva and A. Imparato, Efficiency at Maximum Power of Interacting Molecular Machines, Phys. Rev. Lett. 109, 190602 (2012); Erratum, Phys. Rev. Lett. 110, 149902 (E) (2013).

[11] Y. Apertet, H. Ouerdane, C. Goupil, and Ph. Lecoeur, Efficiency at maximum power of thermally coupled heat engines, Phys. Rev. E 85, 041144 (2012).

[12] R. Wang, J. Wang, J. He, and Y. Ma, Efficiency at maximum power of a heat engine working with a two-level atomic system, Phys. Rev. E 87, 042119 (2013).

[13] S. Q. Sheng and Z. C. Tu, Hidden symmetries and nonlinear constitutive relations for tight-coupling heat engines, New J. Phys. 17, 045013 (2015).

[14] Y. Wang, Optimizing work output for finite-sized heat reservoirs: Beyond linear response, Phys. Rev. E 93, 012120 (2016).

[15] F. Angulo-Brown, An ecological optimization criterion for finite-time heat engines, J. Appl. Phys. 69, 7465 (1991).

[16] A. Calvo Hernández, A. Medina, J. M. M. Roco, J. A. White, and S. Velasco, Unified optimization criterion for energy converters, Phys. Rev. E 63, 037102 (2001).

[17] J. W. Stucki, The optimal efficiency and the economic degrees of coupling of oxidative phosphorylation, Eur. J. Biochem. 109, 269283 (1980).

[18] R. Long and W. Liu, Unified trade-off optimization for general heat devices with nonisothermal processes, Phys. Rev. E 91, 042127 (2015).

[19] V. Singh and R. S. Johal, Feynman's Ratchet and Pawl with ecological criterion: Optimal performance versus estimation with prior information, Entropy 19, 576 (2017).

[20] I. Iyyappan and M. Ponmurugan, Thermoelectric energy converters under a trade-off figure of merit with broken time-reversal symmetry, J. Stat. Mech. (2017) 093207.

[21] M. Bauer, K. Brandner, and U. Seifert, Optimal performance of periodically driven, stochastic heat engines under limited control, Phys. Rev. E 93, 042112 (2016).

[22] K. Proesmans, Y. Dreher, M. Gavrilov, J. Bechhoefer, and C. Van den Broeck, Brownian Duet: A Novel Tale of Thermodynamic Efficiency, Phys. Rev. X 6, 041010 (2016).

[23] K. Funo and M. Ueda, Work Fluctuation-Dissipation TradeOff in Heat Engines, Phys. Rev. Lett. 115, 260601 (2015).

[24] I. Reyes-Ramírez, J. Gonzalez-Ayala, A. Calvo Hernández, and M. Santillán, Local-stability analysis of a lowdissipation heat engine working at maximum power output, Phys. Rev. E 96, 042128 (2017).

[25] J. Gonzalez-Ayala, M. Santillán, I. Reyes-Ramírez, and A. Calvo Hernández, Link between optimization and local stability of a low dissipation heat engine: Dynamic and energetic behaviors, Phys. Rev. E 98, 032142 (2018).

[26] M. Esposito, R. Kawai, K. Lindenberg, and C. Van den Broeck, Efficiency at maximum power of low-dissipation Carnot engines, Phys. Rev. Lett. 105, 150603 (2010).

[27] A. Calvo Hernández, A. Medina, and J. M. M. Roco, Time, entropy generation, and optimization in low-dissipation heat devices, New J. Phys. 17, 075011 (2015). 
[28] J. Gonzalez-Ayala, A. Medina, J. M. M. Roco, and A. Calvo Hernández, Irreversible and endoreversible behaviors of the LD-model for heat devices: The role of the time constraints and symmetries on the performance at maximum $\chi$ figure of merit, J. Stat. Mech. (2016) 073202.

[29] J. Guo, J. Wang, Y. Wang, and J. Chen, Efficiency of two-level weak dissipation quantum Carnot engines at the maximum power, J. Appl. Phys. 113, 143510 (2013).

[30] V. Holubec and R. Artem, Efficiency at and near maximum power of low-dissipation heat engines, Phys. Rev. E 92, 052125 (2015).

[31] J. Gonzalez-Ayala, A. Calvo Hernández, and J. M. M. Roco, From maximum power to a trade-off optimization of lowdissipation heat engines: Influence of control parameters and the role of entropy generation, Phys. Rev. E 95, 022131 (2017).

[32] J. Guo, L. Cai, H. Yang, and B. Lin, Performance characteristics and parametric optimizations of a weak dissipative pumped thermal electricity storage system, Energy Conversion and Management 157, 527 (2017).

[33] Y. H. Ma, D. Xu, H. Dong, and C.P. Sun, Universal constraint for efficiency and power of a low-dissipation heat engine, Phys. Rev. E 98, 042112 (2018).

[34] K. Sekimoto and S. Sasa, Complementarity relation for irreversible process derived from stochastic energetics, J. Phys. Soc. Jpn. 66, 3326 (1997).

[35] Z. C. Tu, Stochastic heat engine with the consideration of inertial effects and shortcuts to adiabaticity, Phys. Rev. E 89, 052148 (2014).

[36] V. Holubec, An exactly solvable model of a stochastic heat engine: Optimization of power, power fluctuations and efficiency, J. Stat. Mech. (2014) P05022.

[37] Y. Izumida, K. Okuda, J. M. M. Roco, and A. Calvo Hernández, Heat devices in nonlinear irreversible thermodynamics, Phys. Rev. E 91, 052140 (2015).

[38] P. R. Zulkowski and M. R. DeWeese, Optimal protocols for slowly driven quantum systems, Phys. Rev. E 92, 032113 (2015).

[39] R. S. Johal, Heat engines at optimal power: Low-dissipation versus endoreversible model, Phys. Rev. E 96, 012151 (2017).

[40] V. Singh and R. S. Johal, Feynman-Smoluchowski engine at high temperatures and the role of the constraints, J. Stat. Mech. (2018) 073205.

[41] J. Gonzalez-Ayala, J. Guo, A. Medina, J. M. M. Roco, and A. Calvo Hernández, companion paper, Optimization induced by stability and the role of limited control near an steady state, Phys. Rev. E 100, 062128 (2019).
[42] K. Deb, Multi-Objective Optimization Using Evolutionary Algorithms (John Wiley \& Sons, Inc., New York, 2001).

[43] S. Kullback and R. A. Leibler, On information and sufficiency, Ann. Math. Stat. 22, 79 (1951).

[44] S. H. Strogatz, Nonlinear Dynamics and Chaos: With Applications to Physics, Biology, Chemistry, and Engineering (Westview Press, Boulder, 2014).

[45] P. E. Kloeden and E. Platen, Numerical Solution of Stochastic Differential Equations (Springer, Berlin 1992).

[46] By matching entropy changes in both models $\Sigma_{h} / t_{h}=m C_{V} \ln \left[r_{p}^{-(1-\gamma) / \gamma}+\eta_{c}^{-1}\left(1-r_{p}^{-(1-\gamma) / \gamma}\right)\right]$ and $\Sigma_{c} / t_{c}=$ $m C_{V} \ln \left[r_{p}^{(1-\gamma) / \gamma}-\eta_{t}\left(r_{p}^{(1-\gamma) / \gamma}-1\right)\right]$, where $m$ is the working fluid mass, $C_{V}$ is the heat capacity at constant volume, $\gamma \equiv C_{p} / C_{V}$ is the adiabatic factor, $r_{p}$ is the pressure ratio, and $\left(\eta_{c}, \eta_{t}\right)$ are the isentropic efficiencies of the compressor and turbine, respectively. External perturbations on this device arise, for instance, if the turbine is combined with a solar collector and fluctuations in the direct solar irradiance due to meteorological conditions (cloudy days induce a power law distribution).

[47] V. Blickle and C. Bechinger, Realization of a micrometresized stochastic heat engine, Nat. Phys. 8, 143 (2012).

[48] I. A. Martínez, É. Roldán, L. Dinis, D. Petrov, J. M. R. Parrondo, and R. A. Rica, Brownian Carnot engine, Nat. Phys. 12, 67 (2015).

[49] T. Shinjo, T. Okuno, R. Hassdorf, K. Shigeto, and T. Ono, Magnetic vortex core observation in circular dots of permalloy, Science 289, 930 (2000).

[50] G. Hrkac, P. S. Keatley, M. T. Bryan, and K. Butler, Magnetic vortex oscillators, J. Phys. D 48, 453001 (2015).

[51] J.L. England, Dissipative adaptation in driven selfassembly, Nat. Nanotechnol. 10, 919 (2015).

[52] N. Perunov, R. A. Marsland, and J. L. England, Statistical Physics of Adaptation, Phys. Rev. X 6, 021036 (2016).

[53] R. Rao and M. Esposito, Nonequilibrium Thermodynamics of Chemical Reaction Networks: Wisdom from Stochastic Thermodynamics, Phys. Rev. X 6, 041064 (2016).

[54] T. J. Kobayashi and Y. Sughiyama, Stochastic and information-thermodynamic structures of population dynamics in a fluctuating environment, Phys. Rev. E 96, 012402 (2017).

[55] N. Perunov, R. A. Marsland, and J. L. England, Statistical Physics of Adaptation, Phys. Rev. X 6, 021036 (2016).

[56] T. E. Ouldridge, C. C. Govern, and P. R. ten Wolde, Thermodynamics of Computational Copying in Biochemical Systems, Phys. Rev. X 7, 021004 (2017).

[57] D. Helbing and T. Vicsek, Optimal self-organization, New J. Phys. 1, 13.113.17 (1999). 